\title{
3D Interactive Visualization Method of Urban Waterlogging Based on Cesium
}

\author{
Xu Zhang a, b, c , Wei Zhou ${ }^{\text {a, b, c }}$, Jie Shen ${ }^{\text {a, b, c } * \text {, Lukáš Herman }}{ }^{\text {d }}$, Yixian Du ${ }^{\text {a, b, c }}$ \\ ${ }^{a}$ Key Laboratory of Virtual Geographic Environment of Ministry of Education, Nanjing Normal University, Nanjing 210023, China; \\ zx1065069013@163.com(X.Z.),weizhou@njnu.edu.cn(W.Z.), shenjie@njnu.edu.cn(J.S.),yixiandu@163.com(Y.D.) \\ ${ }^{b}$ Jiangsu Centre for Collaborative Innovation in Geographic Information Resource Development and Application, Nanjing 210023 , \\ China. \\ ${ }^{c}$ School of Geography Science, Nanjing Normal University, Nanjing 210023, China. \\ d Department of Geography, Faculty of Science, Masaryk University, Brno, Czech Republic; herman.lu@mail.muni.cz (L.H.) \\ * Corresponding author
}

Keywords: Urban waterlogging, Flood, Visualization, Cesium, SWMM

\begin{abstract}
:
Urban waterlogging, as a common natural disaster in China, seriously restricted the development of society. Nowadays, while the computer technology is developing continuously, the urban waterlogging model is also constantly improved. These models can simulate the process of urban waterlogging, but the simulation results are not intuitive. So it is difficult for users to understand how the model works. Therefore, it is important to find a way to show the simulation results so that people can see the waterlogging simulation intuitively. Cesium, as a three-dimensional visualization platform, can reproduce the process of the urban waterlogging. It will make sense if we could show the simulation results on the Cesium platform. Nowadays, many studies focus on both urban waterlogging and visualization methods. However, there are fewer studies on the combination of the two, especially the interactive visualization of urban waterlogging under parameter adjustment. Therefore, this paper mainly focuses on urban three-dimensional interactive visualization method based on Cesium.

On the one hand, the three-dimensional visualization of the urban waterlogging simulation facilitates the intuitive expression of the simulation results. Without visualization, the results of the simulation are only some complicated and unintuitive figures for most non-experts. On the other hand, visualization based on the Cesium platform can better adapt to the cross-platform application. It can better meet the needs of different terminal devices of different users for the visualization platform, so that users can obtain the disaster information more accurately, consistently and intuitively. It is conducive for management departments to respond to sudden disasters more quickly and efficiently.
\end{abstract}

This research aims to propose a three-dimensional dynamic interactive visualization method for urban waterlogging. Particularly, we hope to find out how to integrate urban waterlogging model and 3D visualization platform. With this 3D visualization platform, we can combine the advantages of the SWMM (Storm Water Management Model) and Cesium platform. Using this platform, it will be easier and more effective to respond to disasters for the masses and management departments.

The following two issues are resolved in this study: i) How do model parameters affect the urban waterlogging simulation and visualization results? ii) How to integrate SWMM and Cesium 3D visualization platform?

In order to address the above research objectives, we will apply the following methodologies: i) We will analyse the parameters of the SWMM for the urban waterlogging visualization. Under the premise of understanding the development process of the urban waterlogging, we will analyse the modelling principle of the urban waterlogging, the mechanism of each part of the model separately. Then, we will find out the method of determining the model parameters of urban waterlogging and its influence on the simulation visualization results. ii) We will study integration methods of urban waterlogging model and 3D visualization platform. We will analyse the mechanism and process of urban waterlogging. We will also calculate the urban waterlogging process data by the SWMM, and establish a three-dimensional visualization platform by the node.js and Cesium, which can dynamically show the process of urban waterlogging. iii) We will complete the design and implementation of the interactive visualization platform of urban waterlogging. According to the above research, taking the Xianlin Campus of Nanjing Normal University as an example, we will build a dynamic interactive visualization system of urban waterlogging simulation based on Cesium. We will also verify the effectiveness of the system by comparing it with actual flood situation.

With this study, we expect to answer how model parameters affect the urban waterlogging simulation and visualization results. As expected results, we plan to build an interactive visualization system of urban waterlogging simulation based on Cesium, publish the flood calculation results into the 3D scene. This will make urban waterlogging process shown in the 3D scene. This visualization system is designed for different users, including specialists, government and individual. It means that you can use the system easily even if you are non-cartographers or non-IT-specialists. 